\title{
Características de la formación continuada en comunicación científica impartida a profesionales de la salud
}

\author{
Manuel Ángel Calvo-Calvo
}

Introducción. La trascendencia de los nuevos conocimientos sobre salud y la enfermedad obligan a médicos, enfermeros y otros profesionales sanitarios a saber comunicarlos adecuadamente a la comunidad científica y a la opinión pública.

Objetivo. Conocer las características del Programa de Formación en Comunicación Científica impartido a profesionales de la salud entre 2001 y 2011.

Materiales y métodos. Se realizó un estudio observacional, transversal, descriptivo y retrospectivo en el que se cuantificaron y analizaron los contenidos lectivos teóricos y prácticos de todas las ediciones que se han desarrollado de dicho programa, desde 2001 a 2011.

Resultados y conclusiones. En ese periodo se han realizado 19 ediciones del programa de formación, en las que se han impartido 247,25 horas lectivas teóricas y prácticas de formación en comunicación científica, y se ha instruido a 890 profesionales de la salud, mayoritariamente enfermeras. El contenido lectivo del programa se estructuró en cinco áreas: introducción a la comunicación científica, escritura científica, comunicación oral, elaboración de presentaciones audiovisuales y comunicación en formato póster. Casi la mitad de la carga lectiva del programa se empleó en formar para publicar artículos científicos, y el resto, en instruir para comunicar la ciencia en reuniones científicas. Además, desde 2008 se incluyó formación para afrontar el reto de desarrollar la alfabetización mediática de los profesionales sanitarios. Por la demanda de formación y la importancia de comunicar la ciencia, debería ofertarse formación en comunicación científica en grado y posgrado, y dedicar más contenidos a la alfabetización mediática.

Palabras clave. Comunicación científica. Divulgación científica. Formación continuada. Profesionales de la salud.

Departamento de Enfermería. Facultad de Enfermería, Fisioterapia y Podología. Universidad de Sevilla. Sevilla, España.

Correspondencia: Prof. Manuel Ángel Calvo Calvo. Departamento de Enfermería. Facultad de Enfermería, Fisioterapia y Podología. Universidad de Sevilla. Avda. Sánchez Pizjuán, s/n. E-41009 Sevilla.

E-mail: macalvo@us.es

Conflicto de intereses: No declarado.

Conflict of interests: None declared.

(c) 2013 FEM

\section{Characteristics of continued training in scientific communication provided to health professionals}

Introduction. The importance of new knowledge in health and disease requires doctors, nurses and other health professionals to know how to properly communicate this knowledge to the scientific community and the public.

Aim. To understand the characteristics of the Training Programme in Scientific Communication taught to health professionals between 2001 and 2011.

Materials and methods. To this end, we performed an observational, cross-sectional, descriptive and retrospective study that quantified and analysed the contents of theoretical and practical lessons of all rounds taught in this programme from 2001 to 2011.

Results and conclusions. According to the results, 19 rounds of the training programme were carried out, which imparted 247.25 hours of theoretical and practical lessons on scientific communication to 890 health professionals, mostly nurses. The program's lessons were structured into five areas: introduction to scientific communication, scientific writing, oral communication, preparation of audio-visual presentations, and poster format communication. Almost half the workload of the programme was used to provide training on publishing scientific articles; the rest provided instruction on how to communicate knowledge in scientific meetings. Since 2008, training has also been included to address the challenge of developing media literacy in health professionals. Due to the demand for training and the importance of communicating science, training should be offered in scientific communication at undergraduate and graduate level, and more content should be devoted to media literacy.

Key words. Continuing education. Health personnel. Information dissemination. Information science. 


\section{Introducción}

La comunicación científica tiene como objeto la transmisión pública de la ciencia, del nuevo conocimiento generado, y se desarrolla en dos ámbitos o fases: la diseminación de la ciencia entre científicos $\mathrm{y}$, posteriormente, su difusión y divulgación a la opinión pública [1,2].

Concretamente, la comunicación de la ciencia entre los propios científicos de una disciplina y de la comunidad científica, a la que se denomina comunicación científica especializada, transmite nuevos descubrimientos entre especialistas de una disciplina y también al resto de la comunidad científica, para que esos conocimientos sean conocidos, comprendidos, puestos en práctica, comprobados y evaluados $[3,4]$.

Comunicar la ciencia producida en cualquier disciplina científica es obligación, función y responsabilidad de los investigadores y especialistas que generan los nuevos conocimientos e ideas [5,6], lo que posibilita que esos generadores de conocimiento se conviertan en protagonistas de la construcción del conocimiento [7].

Esa obligación de comunicar la ciencia es aún más exigible en las disciplinas de las ciencias de la salud, sobre todo por la apelación a la responsabilidad social, dada la importancia y consecuencias que para la sociedad tienen los nuevos conocimientos y tecnologías generadas en el área de salud. Esto obliga inexcusablemente a médicos, enfermeros y demás profesionales de la salud que investigan, a transmitir a sus colegas, a las disciplinas afines y a la opinión pública los nuevos conocimientos científicos y técnicos referidos a la salud, la enfermedad y los procesos que se relacionan con esos estados [8].

Los nuevos conocimientos deben comunicarse con eficacia, es decir, de tal modo que lleguen al público interesado, en el formato y lenguaje adecuados, y que sean entendidos y comprendidos por el público destinatario. Por ello es necesario que los profesionales de la salud posean habilidades en comunicación científica que les permitan elaborar correctamente su producción científica e intelectual, para comunicarla eficazmente al resto de la comunidad científica y también a la opinión pública [3].

Sin embargo, la posesión de habilidades en comunicación científica no depende sólo del talento individual o innato del autor científico, en este caso de los profesionales sanitarios, sino que requiere formación, dedicación y disciplina por parte del productor científico [9].

Ocurre que se desconoce cómo se forman los profesionales de la salud en comunicación científi- ca, pues son muy escasos los estudios que informan sobre este asunto. Por esos estudios, se conocen algunas experiencias de formación en comunicación científica en estudios de ciencias fuera de España $[10,11]$ y también se sabe que los médicos residentes (MIR) que se forman en España reciben una formación específica y común para todas las especialidades que incluye metodología científica y estadística, comunicación científica y lectura crítica de la bibliografía [12].

Ante las escasas experiencias formativas en comunicación científica, desarrolladas para profesionales de la salud, en el año 2001 se concibió el Programa de Formación en Comunicación Científica (PFCC), dirigido a profesionales de la salud. Este PFCC, hasta 2011, se ha impartido a estos profesionales en sucesivas ediciones por encargo de la Sociedad Española de Enfermería Nefrológica (SEDEN), del Foro de Enfermería Nefrológica de Valencia (FENV) y de los Hospitales Universitarios Virgen del Rocío, Virgen Macarena y Valme, todos ellos de Sevilla.

La SEDEN y el FENV son sociedades científicas que agrupan respectivamente a las enfermeras españolas y valencianas dedicadas a la enfermería nefrológica, y los tres hospitales reseñados están radicados en Sevilla (España), pertenecen al sistema público de salud de la Comunidad Autónoma de Andalucía (Servicio Andaluz de Salud) y dan cobertura asistencial a casi la totalidad de la población del Área Sanitaria de Sevilla.

Cabe resaltar que en el Hospital Universitario Virgen Macarena, el PFCC también sirvió para impartir la formación específica que los MIR de dicho hospital debían recibir en comunicación científica y lectura crítica de la literatura científica.

Con las sucesivas ediciones que en sesiones presenciales se han impartido de este PFCC, se pretendía desarrollar los conocimientos y habilidades necesarias que permitiesen a los profesionales de la salud comunicar adecuadamente los resultados de sus investigaciones al resto de la comunidad científica, sobre todo a través de la publicación de artículos en revistas científicas o mediante comunicaciones en formato oral o póster en congresos, sin olvidar las posibilidades que en divulgación científica ofrecen los tradicionales y nuevos medios de comunicación social. En consecuencia, esta formación se enmarcaba dentro de la formación continua o permanente, dado que se pretendía formar a profesionales en activo que necesitaban y pretendían mejorar determinados conocimientos, habilidades y actitudes para su desarrollo personal y el de sus respectivas profesiones [13]. 
Para cumplir esos cometidos, el contenido lectivo de este PFCC se estructuró en cinco grandes áreas temáticas, que a su vez se desarrollaron en distintas unidades teóricas y prácticas, y que fueron las siguientes: introducción a la comunicación científica, escritura y redacción de artículos originales y científicos, comunicación oral para reuniones científicas, presentaciones audiovisuales para apoyar una exposición oral, y comunicación en formato póster.

Este contenido lectivo del PFCC fue acreditado por la Agencia de Calidad Sanitaria de Andalucía (Junta de Andalucía), cuando se impartió en los hospitales públicos de Sevilla, y por la Comisión de Formación Continuada de las Profesiones Sanitarias, de la Comunidad de Madrid, cuando se impartió para la SEDEN.

Las cinco áreas temáticas en las que se estructuró el PFCC sirvieron para definir las características de dicho programa, pues en cada edición que se impartió se incluyeron los contenidos teóricos y prácticos de aquellas áreas que en cada momento demandaban los responsables de investigación y formación de las organizaciones a las que se impartía dicha formación. Igualmente, el mayor o menor número de horas lectivas dedicadas a impartir cada área temática en las sucesivas ediciones del PFCC también se adaptaba en función de esas necesidades de formación en comunicación científica detectadas en cada momento.

En consecuencia, analizando aquellas áreas temáticas que se impartieron en las sucesivas ediciones del PFCC y el número de horas o carga lectiva que se dedicó a cada una de ellas, pueden conocerse las características de la formación sobre comunicación científica que han recibido los profesionales de la salud a los que se impartió dicho programa.

Por ello, el objetivo general de este trabajo fue conocer las características de la formación en comunicación científica que se impartió a profesionales de la salud mediante el PFCC, entre 2001 y 2011, en función de las demandas de formación de las instituciones que solicitaron ediciones de dicho programa. En consonancia con ese objetivo general, en este trabajo se plantearon los siguientes objetivos específicos:

- Conocer el número de profesionales de la salud que se han formado con el PFCC desde 2001 a 2011, su sexo y profesión que ejercen.

- Describir el contenido lectivo teórico y práctico de las áreas temáticas en las que se estructuró el PFCC, así como el método seguido para desarrollar dicho contenido.

- Averiguar la carga lectiva teórica y práctica impartida en comunicación científica mediante di- cho programa de formación en el periodo estudiado.

- Conocer y analizar las opiniones del alumnado asistente a las diferentes ediciones del PFCC.

\section{Materiales y métodos}

Dados los objetivos de este trabajo se realizó un estudio observacional, transversal, descriptivo y retrospectivo, en el que se estudiaron las 19 ediciones o cursos que de este PFCC se han realizado desde que comenzó a impartirse en 2001 hasta el 2011.

Para ello, se estudiaron las siguientes variables: número de cursos programados por año, año en que se impartieron, número de alumnos que se han formado con ese programa y profesión que ejercen, áreas temáticas impartidas en cada edición del PFCC y número de horas lectivas teóricas y prácticas que se dedicó a desarrollar cada área temática.

Para obtener los datos de esas variables se revisaron los cronogramas y los contenidos teóricos y prácticos de todas las ediciones que del PFCC se programaron en todo el periodo estudiado, y se cuantificaron las horas lectivas teóricas y prácticas que se dedicaron a cada una de las cinco áreas temáticas del PFCC, en el total de ediciones programadas.

De estas variables estudiadas se obtuvieron su frecuencia absoluta y porcentajes, y de las cuantitativas, se calculó además la media y la desviación estándar.

En la última sesión de las ediciones del PFCC, excepto la impartida al FENV, se aplicó un cuestionario estructurado, autoadministrado, voluntario y anónimo, para conocer la opinión del alumnado. Tras una primera parte donde se preguntaba la edad, sexo y profesión, se continuaba con siete preguntas cerradas y dos abiertas. En las preguntas 3 a 7 se pedía al alumnado que las calificara de 0 a 10 puntos, y de ellas se obtuvo la media aritmética. De las preguntas 8 y 9, que eran abiertas, se realizó un análisis de contenido (Tabla I).

Los datos cuantitativos se introdujeron en el programa estadístico PASW Statistics 18 para su correspondiente procesamiento y, posteriormente, se clasificaron y tabularon para facilitar su valoración e interpretación.

\section{Resultados}

En las 19 ediciones del PFCC que se han desarrollado entre 2001 y 2011, se han formado en comunicación científica 890 profesionales de la salud, de 
Tabla I. Cuestionario de evaluación para el alumnado del Programa de Formación en Comunicación Científica.

En general, ¿este curso ha satisfecho sus expectativas? a

¿El curso le ha sido de utilidad? a

Evalúe el contenido teórico b

Evalúe los ejercicios prácticos realizados ${ }^{b}$

¿Cuál es su grado de satisfacción por haber asistido al curso? b

Evalúe al docente ${ }^{b}$

Evalúe el método seguido para impartir el curso ${ }^{b}$

Señale los aspectos positivos del curso

Señale los aspectos negativos del curso

a Respuesta: sí / parcialmente / no; b Respuesta: de 0 a 10 puntos.

los cuales $680(76,4 \%)$ eran mujeres y $210(23,6 \%)$, hombres.

De esos 890 profesionales formados en comunicación científica, $730(82,02 \%)$ eran enfermeras y $101(11,35 \%)$, médicos. A otras profesiones de la salud, como farmacéuticos, fisioterapeutas, odontólogos, psicólogos, etc., pertenecían 38 (4,27\%) profesionales, y de $21(2,36 \%)$ asistentes al PFCC no consta su profesión.

Del total de profesionales formados mediante el PFCC, 533 (59,89\%) pertenecían a la SEDEN, 279 $(31,35 \%)$ a los tres hospitales universitarios de Sevilla y $78(8,76 \%)$ al FENV.

De las 730 enfermeras que recibieron formación, 611 (84\%) pertenecían a sociedades científicas (SEDEN y FENV) y 119 (16\%) a los hospitales sevillanos. De los 101 (11,35\%) médicos que se han formado con el PFCC, 99 (11,12\%) pertenecían al Hospital Universitario Virgen Macarena, de los cuales $39(4,38 \%)$ eran facultativos especialistas y $60(6,74 \%)$ eran MIR. Los otros $2(0,23 \%)$ médicos que asistieron a alguna edición del PFCC pertenecían al Hospital Universitario de Valme.

En la tabla II se describe el contenido lectivo teórico y práctico, estructurado en cinco áreas temáticas, que podía impartirse en cada edición del PFCC, según la demanda planteada por las entidades interesadas en recibir alguna edición del PFCC.

El método para desarrollar cada curso impartido del PFCC consistía en presentar por el docente los contenidos teóricos mediante la técnica de explica- ción oral, apoyada con una presentación audiovisual, facilitándose y promoviéndose en todo momento la interacción y cooperación entre alumnos y docente.

En las unidades prácticas, el alumnado trabajaba en grupos de no más de cinco componentes y realizaba ejercicios prácticos relacionados con la teoría expuesta anteriormente. Una vez finalizado el ejercicio práctico, todos los miembros del grupo exponían oral y públicamente al resto del alumnado y al docente los resultados o conclusiones obtenidas, acompañado de una presentación audiovisual cuando interesaba. Tras esa exposición se producía un intercambio de ideas y opiniones, se aclaraban dudas y se corregían errores o faltas.

Respecto a la carga lectiva teórica y práctica de las 19 ediciones desarrolladas del PFCC, se impartieron un total de 247,25 horas lectivas de formación en comunicación científica a profesionales de la salud, de las cuales 146 (59,05\%) horas fueron de carácter teórico y 101,25 (40,95\%) horas fueron prácticas (Tabla III).

Sobre la carga lectiva de las áreas temáticas, del total de las 247,25 horas lectivas impartidas con el PFCC, se dedicaron 111,75 (45,2\%) horas al área II, es decir, a formar en escritura y redacción científica (Tabla III). El resto de la carga lectiva del PFCC, es decir, 124,5 (50,35\%) horas, se dedicó a formar en las áreas de comunicación oral, presentaciones audiovisuales y comunicación en formato póster ( $\mathrm{Ta}$ bla III). Del total de las horas lectivas de cada área temática, a contenidos prácticos se dedicaron las siguientes horas: el 48,13\% a comunicación oral, el $45 \%$ a elaboración de presentaciones audiovisuales, el $40,72 \%$ a escritura científica y el $35,71 \%$ a elaboración del póster (Tabla III).

De los 890 asistentes a las distintas ediciones del PFCC, 556 (62,47\%) cumplimentaron la encuesta de evaluación, de los que $310(55,75 \%)$ asistieron a cursos de la SEDEN y $246(44,25)$ a cursos impartidos en los tres hospitales públicos de Sevilla.

Las respuestas a las preguntas 1 y 2 del cuestionario de evaluación se exponen en la tabla IV, y las valoraciones medias sobre 10, realizadas por el alumnado asistente al PFCC, a las preguntas 3 a 7 fueron: 8,3 al contenido teórico, 7,85 a los ejercicios prácticos, 8,42 al grado de satisfacción por asistir al curso, 9,1 al docente y 8,7 al método seguido para impartir el curso.

En síntesis, los aspectos positivos del curso (pregunta 8) más señalados por los encuestados son el método empleado y los contenidos teóricos y prácticos desarrollados: 'curso muy útil por su buen diseño y contenidos', 'el temario ha sido adecuado, 'el 
Tabla II. Contenido lectivo teórico y práctico de las áreas temáticas del Programa de Formación en Comunicación Científica.

\begin{tabular}{|c|c|c|}
\hline & Contenidos teóricos & Contenidos prácticos \\
\hline $\begin{array}{l}\text { Área I. Introducción } \\
\text { a la comunicación } \\
\text { científica }\end{array}$ & $\begin{array}{l}\text { Método científico } \\
\text { Definición e importancia de la comunicación científica de los resultados } \\
\text { de la investigación } \\
\text { Informe de investigación y clases } \\
\text { Literatura científica (primaria, secundaria y gris) y sus características } \\
\text { Estilo de la redacción científica }\end{array}$ & \\
\hline $\begin{array}{l}\text { Área III. } \\
\text { Comunicación oral }\end{array}$ & $\begin{array}{l}\text { Organización de la producción científica en forma de comunicación } \\
\text { oral para reuniones científicas } \\
\text { Hablar en público, estrategias y recursos del orador } \\
\text { Preparación y desarrollo de la conferencia científica y divulgativa }\end{array}$ & $\begin{array}{l}\text { Confección del guión de una comunicación oral o conferencia de } \\
\text { cinco minutos de duración a partir de un artículo original publicado } \\
\text { Preparación y ensayo de su exposición oral }\end{array}$ \\
\hline $\begin{array}{l}\text { Área IV. Elaboración } \\
\text { de presentaciones } \\
\text { audiovisuales }\end{array}$ & $\begin{array}{l}\text { Presentación audiovisual y su confección mediante programas informáticos } \\
\text { Diseño de diapositivas y presentaciones audiovisuales útiles y eficaces } \\
\text { para apoyar una exposición oral } \\
\text { Viejos y nuevos medios para comunicar la ciencia }\end{array}$ & $\begin{array}{l}\text { Elaboración de una presentación audiovisual con Microsoft Office Power } \\
\text { Point para apoyar la comunicación oral } \\
\text { Exposición pública de la comunicación y de la presentación audiovisual } \\
\text { al resto del alumnado }\end{array}$ \\
\hline $\begin{array}{l}\text { Área V. Comunicación } \\
\text { en formato póster }\end{array}$ & $\begin{array}{l}\text { Póster científico y sus elementos. Organización de la producción } \\
\text { científica en forma de póster para reuniones cientificas }\end{array}$ & $\begin{array}{l}\text { Elaboración de un póster mediante Microsoft Office Power Point } \\
\text { Exposición oral ante el resto del alumnado }\end{array}$ \\
\hline
\end{tabular}

curso me ha aportado mucho, sobre todo en temas tan importantes como escribir un artículo o hablar en público.' También señalan positivamente el desarrollo de los contenidos por parte del docente: 'la claridad,' 'sencillez en la exposición,' 'buena presentación' y 'preparación' del docente, y 'el apoyo audiovisual usado' para presentar los contenidos. La entrega por el docente de las unidades teóricas impartidas por escrito y de documentación complementaria para las prácticas también es muy bien valorada por el alumnado.

Como punto débil del curso más señalado (pregunta 9), destaca la falta de tiempo para profundizar más sobre los temas teóricos y para realizar más ejercicios prácticos: 'debería repetirse este curso, pero con más horas de teoría y práctica', 'el curso debería tener más horas para hacer más ejercicios prácticos'.

\section{Discusión}

Dado que el objetivo general de este trabajo fue conocer las características de la formación en comunicación científica impartida a profesionales de la salud entre 2001 y 2011, se puede afirmar que, a la luz de los resultados, una primera característica destacable del PFCC fue el gran interés de los profesionales de las organizaciones demandantes de dicho programa en adquirir habilidades para comunicar la ciencia, como demuestra el número de ediciones desarrolladas del PFCC, las horas de formación impartidas y el elevado número de profesionales de la salud asistente.

Otra característica que destaca es que con este PFCC para profesionales de la salud se han formado mayoritariamente enfermeras, dada la superior asistencia de éstas en comparación con los médicos y otros profesionales de la salud [14]. Esta superior asistencia de enfermeras creemos que se relacionaba directamente con los ámbitos institucionales en los que se ha impartido el PFCC, ya que casi el 70\% de los asistentes a los 19 cursos celebrados pertenecían a la SEDEN y al FENV, sociedades científicas con un elevado número de socios pero que acogen exclusivamente a enfermeras.

Igualmente, este PFCC se ha caracterizado por la gran importancia que se ha dado a los contenidos prácticos a la hora de diseñar sus diferentes ediciones, puesto que en todas las áreas temáticas del 
Tabla III. Número de horas lectivas teóricas y prácticas en comunicación científica impartidas por área temática.

\begin{tabular}{|c|c|c|c|c|c|c|}
\hline & \multicolumn{2}{|c|}{$\begin{array}{l}\text { Horas teóricas } \\
\text { (total: } 146,00 \mathrm{~h} \text { ) }\end{array}$} & \multicolumn{2}{|c|}{$\begin{array}{l}\text { Horas prácticas } \\
\text { (total: } 101,25 \mathrm{~h} \text { ) }\end{array}$} & \multicolumn{2}{|c|}{$\begin{array}{l}\text { Horas totales } \\
\text { (total: } 247,25 \mathrm{~h} \text { ) }\end{array}$} \\
\hline & $n$ & $\%$ & $n$ & $\%$ & $n$ & $\%$ \\
\hline I. Introducción a la comunicación científica & 11,00 & 7,53 & 0,00 & 0,00 & 11,00 & 4,45 \\
\hline II. Escritura científica & 66,25 & 45,38 & 45,50 & 44,94 & 111,75 & 45,20 \\
\hline III. Comunicación oral & 27,75 & 19,01 & 25,75 & 25,43 & 53,50 & 21,64 \\
\hline IV. Presentaciones audiovisuales & 27,50 & 18,83 & 22,50 & 22,22 & 50,00 & 20,22 \\
\hline V. Comunicación formato póster & 13,50 & 9,50 & 7,50 & 7,41 & 21,00 & 8,49 \\
\hline
\end{tabular}

PFCC se programaron ejercicios prácticos, excepto en el área I, donde no eran necesarios. Consecuencia de ello fue que en el total de las ediciones impartidas del PFCC en los 11 años, la carga lectiva teórica sólo fue algo superior a la práctica, dado que por cada hora impartida de teoría se dispuso de 41,6 minutos para realizar ejercicios prácticos ( $\mathrm{Ta}$ bla III). No obstante, el alumnado asistente a las distintas ediciones del PFCC señala que se debería dedicar más tiempo a los ejercicios prácticos o profundizar más en ellos, cuestión que deberá tenerse en cuenta en el diseño de los contenidos de futuras ediciones.

Otra característica importante del PFCC fue que su contenido teórico y práctico, estructurado en las cinco áreas temáticas (Tabla II), pretendía abarcar la formación en todas las modalidades de la comunicación científica, lo cual fue muy bien valorado por el alumnado. Así, los contenidos dedicados a enseñar a escribir artículos científicos, a elaborar y presentar comunicaciones orales o en formato póster para congresos científicos, estaban dirigidos a formar a los profesionales de la salud en comunicación científica especializada. Igualmente, los temas sobre redacción de artículos divulgativos, la conferencia divulgativa y los nuevos medios para comunicar la ciencia pretendían formar a los profesionales de la salud en divulgación científica.

No obstante, en función de la carga lectiva que tuvieron las diferentes áreas temáticas impartidas del PFCC, y dado que solo al área II (escritura científica) se dedicó casi la mitad de sus horas lectivas, se puede señalar que ha sido un programa dirigido sobre todo a aumentar el conocimiento y desarrollar las habilidades de los profesionales de la salud para escribir y publicar artículos en revistas cientí- ficas. Esto, además de obedecer a la mayor demanda de los profesionales por formarse en esa área, puede estar influido también por la idea tradicional de que publicar en revistas científicas es el método formal, más adecuado y válido para comunicar la ciencia, y que mantiene en vigor la vieja idea de que para hacer ciencia, obligatoriamente hay que escribir ciencia.

A las áreas de comunicación oral, elaboración de presentaciones audiovisuales y comunicación en formato póster se les dedicó la mitad de horas teóricas y prácticas del PFCC (Tabla III). Como esas tres áreas en conjunto están dirigidas a desarrollar habilidades para comunicar la ciencia en reuniones científicas como congresos, seminarios o jornadas, se puede afirmar que la otra mitad del PFCC se dedicó a formar a los profesionales de la salud para comunicar la ciencia en reuniones científicas.

En consecuencia, este PFCC se ha caracterizado por suministrar formación en comunicación científica que podríamos denominar 'integral' porque ha dado prácticamente la misma importancia a formar tanto en comunicar la ciencia publicando en revistas científicas como asistiendo a reuniones científicas, característica que también ha sido valorada muy positivamente por el alumnado. Esta formación integral en comunicación científica constituye una característica particular e innovadora de este PFCC porque las escasas experiencias formativas existentes en esta materia no incluyen formación en las tres áreas dedicadas a comunicar la ciencia en reuniones científicas o la incluyen mínimamente [11].

En esas escasas experiencias conocidas de formación en comunicación científica tampoco se ha puesto énfasis en la necesidad de que los comunicadores científicos estén alfabetizados mediáticamen- 
te, es decir, que posean capacidad de acceso a los medios de comunicación para comprender y evaluar críticamente los contenidos mediáticos, y que también adquieran capacidad para crear comunicaciones en una variedad de contextos [15]. Sólo algunos autores hablan de las posibilidades que ofrecen los nuevos medios que surgen con el apogeo de Internet y con la irrupción de la web 2.0 para comunicar la ciencia, dado que posibilitan que cualquier persona con acceso y conocimientos sobre esas nuevas tecnologías pueda convertirse no sólo en consumidora, sino también en creadora de contenidos mediáticos [16,17].

Contrariamente a esa tendencia, el PFCC se ha caracterizado por dedicar espacio al papel de los nuevos medios para comunicar la ciencia y a la necesidad de que los comunicadores científicos estén alfabetizados mediáticamente al introducir el tema 'Viejos y nuevos medios para comunicar la ciencia' en el área IV (Tabla III). Por tanto, en este PFCC se ha entendido la alfabetización mediática de los profesionales de la salud como un reto, dado que en él se han propuesto estrategias para conseguir esa alfabetización. Con ello se pretendía conseguir que los profesionales de la salud fuesen eficaces comunicadores científicos pero también competentes mediáticamente, es decir, que supiesen también difundir y expresarse a través de los viejos y nuevos medios de comunicación, y con capacidad de producción y de creación de contenidos desde la competencia digital. Este objetivo, además de innovador, estaba plenamente justificado porque los profesionales sanitarios, al estar alejados de los entresijos de la industria mediática, conviene que conozcan y aprovechen las potencialidades de los medios de comunicación para diseminar el nuevo conocimiento generado. Por tanto, este propósito de desarrollar las habilidades mediáticas en los profesionales sanitarios fue otra de las características novedosas y un compromiso de este PFCC para afrontar el reto de mejorar y desarrollar la alfabetización mediática de los profesionales sanitarios en esta era digital.

Creemos que a estas características que se han destacado se ha debido el alto grado de satisfacción y de utilidad mostrado por el alumnado por asistir a las ediciones del PFCC, que dicho PFCC fuese parte de la formación de los MIR en el Hospital Virgen Macarena durante tres cursos académicos, y que las distintas ediciones del PFCC hayan sido acreditadas por agencias oficiales de evaluación.

La experiencia de 11 años impartiendo sucesivas ediciones del PFCC permite proponer modificaciones y nuevas estrategias que puedan contribuir a mejorar la calidad de la formación que se imparta
Tabla IV. Evaluación del Programa de Formación en Comunicación Científica por el alumnado: preguntas cerradas 1 y 2 (total: 556 respuestas).

\begin{tabular}{lccc}
\hline & Sí & Parcialmente & No \\
\hline $\begin{array}{l}\text { En general, ¿este curso } \\
\text { ha satisfecho sus expectativas? }\end{array}$ & $508(91,37 \%)$ & $44(7,91 \%)$ & $4(0,72 \%)$ \\
\hline ¿El curso le ha sido de utilidad? & $491(88,31 \%)$ & $62(11,15 \%)$ & $3(0,54 \%)$ \\
\hline
\end{tabular}

en el futuro. En este sentido, dada la emergencia de Internet como nuevo medio para comunicar la ciencia, y sobre todo de la filosofía web 2.0, la red basada en comunidades de usuarios donde éstos interactúan a través de redes sociales, blogs, etc., sería necesario dedicar más contenidos sobre las nuevas posibilidades que ofrecen esas nuevas tecnologías para diseminar y divulgar el conocimiento y la producción científica.

Otra línea de trabajo para mejorar la formación impartida sería incorporar esas nuevas tecnologías de la información también como herramientas metodológicas y docentes en el PFCC, y contemplar el uso de plataformas virtuales de apoyo a la docencia [18]. Con ello se podrían implementar nuevas metodologías docentes, abarcar más contenidos temáticos en comunicación científica, flexibilizar la formación mediante la formación 'a la carta' o 'bajo demanda' donde el alumnado elige materias de su preferencia en las que formarse y no hacer de la presencia del alumnado una obligación para la formación. Estas nuevas posibilidades que aportarían las nuevas tecnologías podrían contribuir además a satisfacer la demanda del alumnado de mayor dedicación y profundización en los contenidos prácticos.

También sería muy interesante evaluar las aportaciones de la formación en comunicación científica, tanto desde la propia percepción de los profesionales como desde la cuantificación de la producción científica de investigadores y entidades que han recibido dicha formación en comunicación científica. Se podría estudiar así si dicha formación generó un aumento de la producción, difusión y divulgación de nuevos conocimientos en los profesionales de la salud y en sus instituciones.

Debido a la casi total ausencia de estudios sobre formación en comunicación científica, este trabajo, además de aportar criterios para mejorar la formación a impartir en comunicación científica en el futuro, puede servir también como guía y orientación a quienes precisen organizar y estructurar alguna actividad de formación en comunicación científica. 
Igualmente, y dada la importancia que tiene la comunicación científica para el desarrollo de los profesionales de la salud, de sus disciplinas y de la ciencia en general, hace aconsejable que esta materia sea impartida en todo el continuo de la formación de los profesionales sanitarios, y que abarca el grado, el posgrado y especialidad, y la formación continua y desarrollo profesional continuo [19].

\section{Bibliografía}

1. Elías C. La 'cultura convergente' y la filosofía Web 2.0 en la reformulación de la comunicación científica en la era del ciberperiodismo. Arbor 2009; 185: 623-34.

2. Martín MJ, Rey J. El concepto de comunicación científica: diseminación, difusión y divulgación científica. In Martín M] Rey J, eds. El papel de los científicos en la comunicación de la ciencia y la tecnología a la sociedad: actitudes, aptitudes e implicación. Madrid: Dirección General de Universidades e Investigación de la Comunidad de Madrid; 2007. p. 36-9.

3. Calvo-Calvo MA. Introducción a la comunicación científica para profesionales de la salud. El artículo original, la comunicación oral, el póster y la presentación audiovisual. 2 ed. Madrid: Sociedad Española de Enfermería Nefrológica; 2005.

4. Pedrós G. La construcción dialéctica del conocimiento y la divulgación científica. Comunicar 2002; 19: 61-5.

5. De Semir V. Metaanálisis: comunicación científica y periodismo científico. Madrid: Fundación Española para la Ciencia y la Tecnología; 2011.

6. Torres-Salinas D, Delgado López-Cózar E. Estrategia para mejorar la difusión de los resultados de investigación con la Web 2.0. El Profesional de la Información 2009; 18: 534-9.

7. Rodríguez-López J. Ciencia y comunicación científica: edición digital y otros fundamentos del libre acceso al conocimiento. El Profesional de la Información 2005; 14: 246-54.
8. Rodríguez-Gázquez MA. La necesidad de difundir conocimiento en la disciplina de enfermería. Invest Educ Enferm 2011; 29: 7-8.

9. García JM. Cómo escribir un artículo original para una revista biomédica. Radiología 2011; 53: 209-19.

10. Spektor-Levy O, Eylon B, Scherz Z. Teaching scientific communication skills in science studies: does it make a difference? International Journal of Science and Mathematics Education 2009; 7: 875-903.

11. Scherz Z, Spektor-Levy O, Eylon B. 'Scientific Communication': an instructional program for high-order learning skills and its impact on students' performance. In Boersma K, Goedhart M, De Jong $\mathrm{O}$, Eijkelhof $\mathrm{H}$, eds. Research and the quality of science education. Amsterdam: Springer; 2005. p. 231-43.

12. Puig J. Formación en habilidades complementarias y radiología. Radiología 2011; 53: 220-5.

13. Pineda P, Sarramona J. El nuevo modelo de formación continua en España: balance de un año de cambios. Revista de Educación 2006; 341: 705-36.

14. Calvo-Calvo MA. Características de un programa de formación postgrado en comunicación científica dirigido a enfermeras [abstract]. Actas y Comunicaciones de la XI Conferencia Iberoamericana de Educación en Enfermería. Coímbra, Portugal, 18-24 de septiembre de 2011; 2011; 4 (Suppl 2): 88

15. Silver A. Aproximación europea a la educación en medios: avanzando hacia una sociedad del conocimiento inclusiva. Comunicar 2009; 32: 19-20.

16. Kneipp Sena N. Open archives: caminho alternativo para a comunicação científica. Ciência da Informação 2000; 29: 71-8.

17. Lercher A. Efficiency of scientific communication: a survey of world science. J Am Soc Inf Sci Technol 2010; 61: 2049-60.

18. López-Morales M, Celma-Vicente M, Cano-Caballero MD, Quero-Rufián A, Rodríguez-López MA. Docencia universitaria semipresencial. Experiencia en el uso de la plataforma virtual SWAD. Educ Med 2011; 14: 229-34.

19. Mirón-Canelo JA, Iglesias-De Sena H, Alonso-Sardón M. Valoración de los estudiantes sobre su formación en la facultad de medicina. Educ Med 2011; 14: 221-8. 